\title{
Facial Herpes Simplex Virus Type 1 Infection in a Patient with Multiple Sclerosis on Fingolimod
}

\author{
Racosta Juan Manuel, McEwan Lynn, Kremenchutzky Marcelo Carlos, Morrow Sarah Anne* \\ University of Western Ontario, London, Ontario \\ *Corresponding author: sarah.morrow@lhsc.on.ca
}

Received March 18, 2015; Revised April 15, 2015; Accepted April 23, 2015

\begin{abstract}
Fingolimod is an oral disease-modifying therapy for relapsing multiple sclerosis widely used in Canada as a second-line therapy. Fingolimod increases the risk of infections given its dose-dependent reduction of peripheral lymphocytes, due to the reversible sequestration in lymphoid tissues. We present a case of disseminated facial Herpes Simplex Virus type I infection in a patient using fingolimod, with an atypical distribution of the eruption, likely due to the concomitant exposure to sunlight and application of sunscreen. This case report provides insight about a novel mechanism of disseminationof Herpes Simplex Virus type I, which generally enters damaged epithelium or mucosal surfaces secondary to abrasions or trauma. Since Herpes Simplex Virus type I infection tends to disseminate and is accompanied by visceral involvement in immunosuppressed patients, close surveillance of viral infections and the prompt administration of full-dose antiviral therapy in this population is advised.
\end{abstract}

Keywords: multiple sclerosis, fingolimod; herpes simplex, infection, adverse effect

Cite This Article: Racosta Juan Manuel, McEwan Lynn, Kremenchutzky Marcelo Carlos, and Morrow Sarah Anne, "Facial Herpes Simplex Virus Type 1 Infection in a Patient with Multiple Sclerosis on Fingolimod." American Journal of Medical Case Reports, vol. 3, no. 5 (2015): 144-145. doi: 10.12691/ajmcr-3-5-7.

\section{Background}

Fingolimod is an oral disease-modifying therapy approved in Canada for relapsing multiple sclerosis (MS)as a second-line option or escalating therapy and as a first line therapy in the United States. Fingolimod acts via modulation of the sphingosine-1-phosphate receptor [1]. Due to the reversible sequestration of lymphocytes in lymphoid tissues it produces a dose-dependent reduction of peripheral lymphocyte count to $20-30 \%$ of baseline values, increasing the risk of infections [2].We describe a patient with MS who developed bilateral facial Herpes Simplex (HSV) Type 1 infection during treatment with fingolimod 0.5mg OD.

\section{Case Presentation}

A case of a 45-year-old right handed woman diagnosed with relapsing-remitting multiple sclerosis in 2012, initially treated with interferon $\beta-1$ a subcutaneously, 44ug/t.i.w., was switched to fingolimod because of injection sites adverse reactions. Her past medical history included hypothyroidism treated with levothyroxine. She had good compliance with fingolimod, prior to her current presentation, 29 months after starting fingolimod. She initially noticed left eye discomfort, associated with mild photophobia and tearing, and a lesion at the corner of her eye. She was diagnosed with bacterial conjunctivitis and started treatment with antibiotic eye drops. After a few days a facial eruption developed, characterized by confluent vesicles and a few pustules localized on her cheeks, forehead, nose, jaws and chin bilaterally (Figure 1). There were no signs of facial eczema or other dermatitis. After consultation with an infectious disease specialist, cutaneous herpes was suspected and a swab for immunofluorescence was performed. Herpes Simplex Virus (HSV) type 1 infection was confirmed. She started treatment with famciclovir $500 \mathrm{mg}$ t.i.d. for 1 week with good resolution of the lesions. A brain MRI 2 weeks after onset of her rash was consistent with her MS diagnosis and unchanged since the previous MRI. Previous and current blood work demonstrated lymphopenia since 3 months after starting fingolimod, ranging from 200 to 600 cells $/ \mu \mathrm{L}$, (Normal threshold 1,500 cells $/ \mu \mathrm{L}$ ).

In retrospect, the subject mentioned travel to Florida for a 3 day vacation after the appearance of the first lesion, during which she applied sunscreen to her face, applying it in a circular fashion, which preceded the disseminated rash.

\section{Conclusions}

The immunosuppressive effects of fingolimod require increased awareness of infectious complications. Higher incidence of viral infections caused by Varicella Zoster virus (VZV) and the Influenza virus have been reported in fingolimod-treated patients vs. those given placebo [3]. Due to two fatal VZV infections during the TRANSFORMS trial, all patients without a history of chicken pox or vaccination need to be tested for antibodies to VZV [4]. 


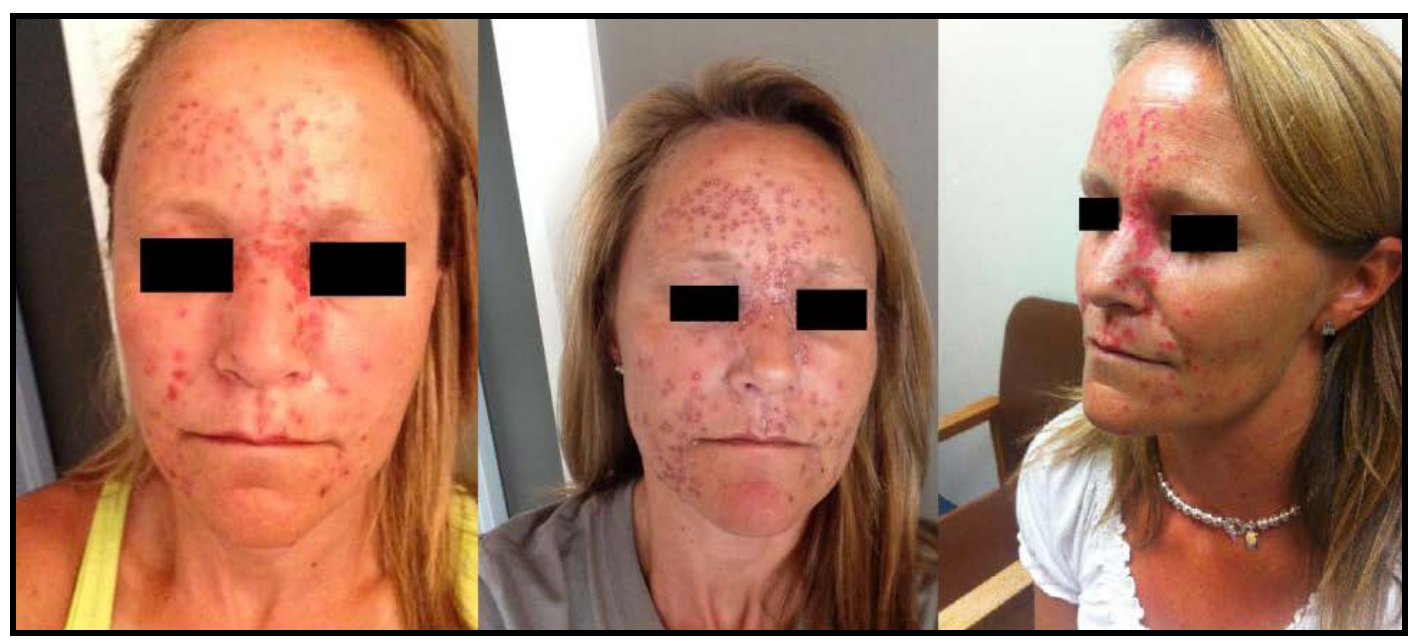

Figure 1. A 45-year-old womanwith a vesicular eruption on forehead, cheeks, nose, jaws and chin. The pictures show the evolution within a 6 weeks period

We present a case of disseminated facial HSV type I infection in a patient with a history of relapsing-remitting multiple sclerosis receiving fingolimod. Since HSV type 1 enters damaged epithelium or mucosal surfaces, secondary to abrasions or trauma, the atypicalfacial distribution of the eruption in this case may have beenfavored by the concomitant exposure to sunlight and the applicationof sunscreen during the symptomatic phase. Similar factors promoting the local spreading of HSV during primary infections, such as shaving, have been previously reported [5].

The use of fingolimod in MS demonstrated to produce decreased lymphocyte counts to $70 \%$ of baseline within 4 hours after the first dose, and approximately 50\% within 8 hours. With continued daily dosing, the lymphocyte count continues decreasing over a 2 -week period, reaching a nadir count of approximately 500 cells $/ \mu \mathrm{L}$ or approximately $30 \%$ of baseline [2]. Our subject had a sustained lymphopenia 3 months after starting fingolimod with levels fluctuating between 200 and 600 cells $/ \mu \mathrm{L}$. Since normal immune response functions are maintained during fingolimod treatment [6], it is thought that fingolimod modulation of lymphocytes egress does not inhibit their effector functions. Regardless, local immune responses that depend on recently activated $\mathrm{T}$ cells can be reduced, as they remain retained in lymphoid tissues. This may increase the risk of infections, including common viral infections such as HSV [7].

To our knowledge, this is the first report of a disseminated facial HSV type 1 infection in a in a patient with MS receiving fingolimod. We believe this unusual adverse reaction to be as a direct result of her immunosuppressive therapy. We support close surveillance of viral infections and the prompt administration of full-dose antiviral therapy in this population, in order to avoid the possibility of serious, potentially fatal, complications.

\section{Consent}

Written informed consent was obtained from the patient for publication of this Case report and the accompanying image. A copy of the written consent is available for review by the Editor of this journal.

\section{Competing Interests}

The authors declare that they have no competing interests.

\section{Authors Contributions}

Racosta JM: study concept and design, drafting of the manuscript.

McEwan L: data adquisition, study concept and design.

Kremenchutzky MC: critical revision of the manuscript for important intellectual content.

Morrow SA: study concept and design, drafting and critical revision of the manuscript for important intellectual content, critical revision of the manuscript, study supervision.

\section{References}

[1] Cohen J.A., Chun J. "Mechanisms of fingolimod's efficacy and adverse effects in multiple sclerosis", Annals of Neurology, 69(5).759-777. May. 2011.

[2] Kappos L., Radue E.W., O'Connor P., Polman C., Hohlfeld R. Calabresi P., et al. "A placebo-controlled trial of oral fingolimod in relapsing multiple sclerosis", New England Journal of Medicine, 362(5).387-401. Feb.2010.

[3] Calabresi P.A., Radue E.W., Goodin D., Jeffery D., Rammohan K.W., Reder A.T., et al. "Safety and efficacy of fingolimod in patients with relapsing-remitting multiple sclerosis (FREEDOMS II): A double-blind, randomised, placebo-controlled, phase 3 trial", Lancet Neurology, 13(6).545-556. Jun.2014.

[4] Khatri B., Barkhof F., Comi G., Hartung H.P., Kappos L., Montalban X., et al. "Comparison of fingolimod with interferon beta-1a in relapsing-remitting multiple sclerosis: A randomised extension of the TRANSFORMS study",Lancet Neurology, 10(6).520-529.Jun.2011.

[5] Maalouf E., Moutran R., Maatouk I. "Disseminated primary HSV2 infection of the face",Dermatology Online Journal, 18(6).15. Jun.2012.

[6] Brinkmann V., Chen S., Feng L., Pinschewer D., Nikolova Z., Hof R.. "FTY720 alters lymphocyte homing and protects allografts without inducing general immunosuppression", Transplantation Proceedings, 33(1-2).530-531. Feb-Mar. 2001.

[7] Pinschewer D.D., Ochsenbein A.F., Odermatt B., Brinkmann V., Hengartner H., Zinkernagel R.M. "FTY720 immunosuppression impairs effector $\mathrm{T}$ cell peripheral homing without affecting induction, expansion, and memory",Journal of Immunology, 164(11).5761-70.Jun. 2000. 